Archive for

Organic Chemistry

Arkivoc 2019, part vi, 174-179

\title{
Methylamine-induced ring opening of 1,3-dithiolium cations
}

\section{Laura G. Sarbu, ${ }^{\text {a }}$ Henning Hopf, ${ }^{b}$ Peter G. Jones, ${ }^{c}$ Lucian G. Bahrin, ${ }^{\text {a,d }}$ and M. Lucian Birsa ${ }^{a *}$}

a Department of Chemistry, "Al. I. Cuza" University of Iasi, 11 Carol I Bv., RO-700 506 Iasi, Romania

${ }^{b}$ Institute of Organic Chemistry, Technical University of Braunschweig, Hagenring 30, D-38106 Braunschweig, Germany

c Institute of Inorganic and Analytical Chemistry, Technical University of Braunschweig, Hagenring 30, D-38106 Braunschweig, Germany

d "Petru Poni" Institute of Macromolecular Chemistry, Aleea Grigore Ghica Vodă 41A, 700487 Iasi, Romania Email: Ibirsa@uaic.ro

\section{Abstract}

An unprecedented retrocyclization of 1,3-dithiolium cations in the presence of methylamine has been discovered that leads to the corresponding dithiocarbamates. X-ray structural analyses of two products confirm the reaction outcome.

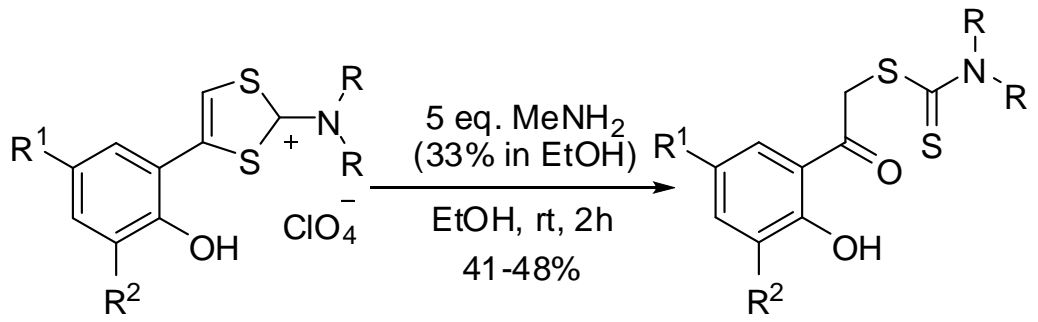

Keywords: Amines, dithiolium cations, dithiocarbamates, imines 


\section{Introduction}

1,3-Dithiolium salts are well known precursors of tetrathiafulvalenes, compounds with wide applications in materials chemistry. ${ }^{1,2}$ However, reviews of their chemical properties make clear that their chemistry is by no means limited to tetrathiafulvalene preparations. ${ }^{3,4}$ The electrophilic properties of the $\mathrm{C}-2$ atom lead to many interesting reactions; for example, the interaction of these cations with nitrogen nucleophiles has usually been exploited for the synthesis of 2-iminochalcogen fulvalenes..$^{5-7}$ However, 1,3-dithiols were reported to undergo ring-opening reactions under various conditions. Thus, the reactions of 4-(2-hydroxyaryl)-2-( $\mathrm{N}, \mathrm{N}$-dialkylamino)1,3-dithiolium salts with sodium sulfide enneahydrate in boiling ethanol provided the corresponding substituted 2-hydroxyacetophenones by carbon disulfide extrusion. ${ }^{8}$ An amine-mediated ring-opening reaction of 2-methylene-1,3-dithioles activated by electron-withdrawing groups was discovered, and a new route to highly substituted thiophenes was developed via the ring opening of 1,3-dithioles and subsequent intramolecular annulation and amine substitution. ${ }^{9}$ Moreover, the ring opening reaction of 1,3-dithiol-2-one systems was reported to be reversible. ${ }^{10}$ Acetylenic amines and sodium sulfide have also been employed in ring opening of 3-azaisatoic anhydride and epoxides. ${ }^{11,12}$

We report here a new reaction, namely the methylamine-induced retrocyclisation of 1,3-dithiolium rings to the corresponding $\mathrm{N}, \mathrm{N}$-dithiocarbamates .

\section{Results and Discussion}

Following previous experiments, in which the treatment of 1,3-dithiolium salts of type 1 with $n$-propylamine and iso-butylamine provided the corresponding 1,3-dithiol-2-imine derivatives, ${ }^{7}$ we extended this study to the reaction of 1,3-dithiolium cations with methylamine. Thus, by treating a suspension of 1,3-dithiolium perchlorates 1a-j in ethanol with excess methylamine ( $33 \%$ in ethanol) a homogeneous solution was obtained from which solid products were precipitated by water (Table 1). Unexpectedly, the spectral analysis of these products indicated the structure of the 2-hydroxyphenacyl dithiocarbamates (2), compounds that were previously obtained by the reaction of the corresponding substituted $\omega$-bromoacetophenones with various salts of dithiocarbamic acids.

The NMR analysis of the crude reaction mixture also indicated the presence of small amounts of the corresponding 2-hydroxyacetophenones along with other unidentified compounds. This is in accordance with our previous findings, whereby the treatment of 1,3-dithiolium salts with sodium sulfide in ethanol under reflux conditions also afforded the corresponding 2-hydroxyacetophenones. ${ }^{8}$ The presence of the hydroxy group was found to control the outcome of that reaction. Consequently, we assume that, in a first step, methylamine attacks the electron-deficient C-2 position of the 1,3-dithiolium ring, and then the ring opening takes place with the formation of a methyl imine intermediate. The formation of the dithiocarbamates requires, in this step, a sulfur nucleophile to replace the methyl imine moiety. Bearing in mind that all these dithiocarbamates were isolated in less than $50 \%$ yield, it is reasonable to assume that the sulfur atom originates from the decomposition of a part of the methyl-imine intermediate, providing the corresponding 2hydroxyacetophenones identified in the crude reaction mixture. Finally, the acid hydrolysis of this mixture provides the oxygen atom of the carbonyl group. 
Table 1. Reactions of 1,3-dithiolium perchlorates (1) with excess methylamine<smiles>[R]c1cc([R2])c(O)c(-c2csc(N([R])[Tl])[s+]2)c1</smiles>

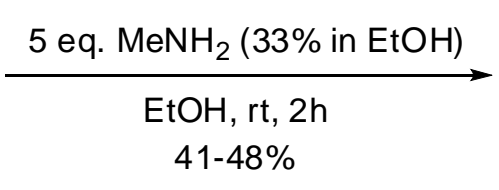<smiles>[R]c1cc([R7])c(O)c(C(=O)CSC(=S)N([R])[Tl])c1</smiles>

\begin{tabular}{ccccccc}
\hline $\mathbf{2}$ & $\mathbf{R}$ & $\mathbf{R}$ & $\mathbf{R}^{\mathbf{1}}$ & $\mathbf{R}^{\mathbf{2}}$ & Yield, \% & M.p., ${ }^{\circ} \mathrm{C}$ (lit. $)^{\text {ref }}$ \\
\hline $\mathbf{a}$ & $\mathrm{CH}_{3}$ & $\mathrm{CH}_{3}$ & $\mathrm{H}$ & $\mathrm{H}$ & 44 & $118-119(119-120)^{13}$ \\
$\mathbf{b}$ & $\mathrm{CH}_{3}$ & $\mathrm{CH}_{3}$ & $\mathrm{CH}_{3}$ & $\mathrm{H}$ & 47 & $151-152(151-152)^{14}$ \\
$\mathbf{c}$ & $\mathrm{CH}_{3}$ & $\mathrm{CH}_{3}$ & $\mathrm{Br}$ & $\mathrm{H}$ & 45 & $185-186(184-185)^{15}$ \\
$\mathbf{d}$ & $\mathrm{CH}_{3}$ & $\mathrm{CH}_{3}$ & $\mathrm{Br}$ & $\mathrm{CH}_{3}$ & 42 & $157-158(157-158)^{16}$ \\
$\mathbf{e}$ & $\mathrm{C}_{2} \mathrm{H}_{5}$ & $\mathrm{C}_{2} \mathrm{H}_{5}$ & $\mathrm{Br}$ & $\mathrm{H}$ & 41 & $121-122(123-124)^{15}$ \\
$\mathbf{f}$ & $\left(\mathrm{CH}_{2}\right)_{4}$ & $\mathrm{CH}_{3}$ & $\mathrm{H}$ & 46 & $139-140(140-141)^{14}$ \\
$\mathbf{g}$ & $\left(\mathrm{CH}_{2}\right)_{4}$ & $\mathrm{CH}_{3}$ & $\mathrm{Br}$ & 47 & $172-173(171-172)^{17}$ \\
$\mathbf{h}$ & $\left(\mathrm{CH}_{2}\right)_{4}$ & $\mathrm{Br}$ & $\mathrm{H}$ & 45 & $156-157(156-157)^{15}$ \\
$\mathbf{i}$ & $\left(\mathrm{CH}_{2}\right)_{5}$ & $\mathrm{CH}_{3}$ & $\mathrm{H}$ & 44 & $134-135(135-136)^{14}$ \\
$\mathbf{j}$ & $\left(\mathrm{CH}_{2}\right)_{5}$ & $\mathrm{CH}_{3}$ & $\mathrm{Br}$ & 48 & $166-167(165-166)^{17}$ \\
\hline
\end{tabular}

This behavior of the 1,3-dithiolium cations towards methylamine has not been reported before. The methyl-imine moiety probably plays an important role for the reaction outcome. This reaction may serve as a model for ring opening of other cyclic cations of this type.

\section{X-Ray analyses}

Molecular structures of dithiocarbamates (2a) and (2b), based on X-ray analyses, are presented in Figure 1 and Figure 2, respectively. Both compounds have an intramolecular hydrogen bond indicated by a dashed line. The molecular structure of $\mathbf{2 a}$ is planar (mean deviation $0.027 \AA$ ) except for the hydrogen atoms at C8, C10 and $\mathrm{C} 11$. The molecular structure of $\mathbf{2} \mathbf{b}$ consists of two planar regions: the left-hand side up to and including $\mathrm{C} 8$, and the right-hand side from C8 to the dimethylamino group. Mean deviations are 0.024 and $0.033 \AA$, respectively, and the interplanar angle is $79.4^{\circ}$. The methyl hydrogens at C10 (for $\mathbf{2 a}$ ), C10 and C11 (for $\mathbf{2 b}$ ) were disordered over a hexagon of half-occupied positions. 


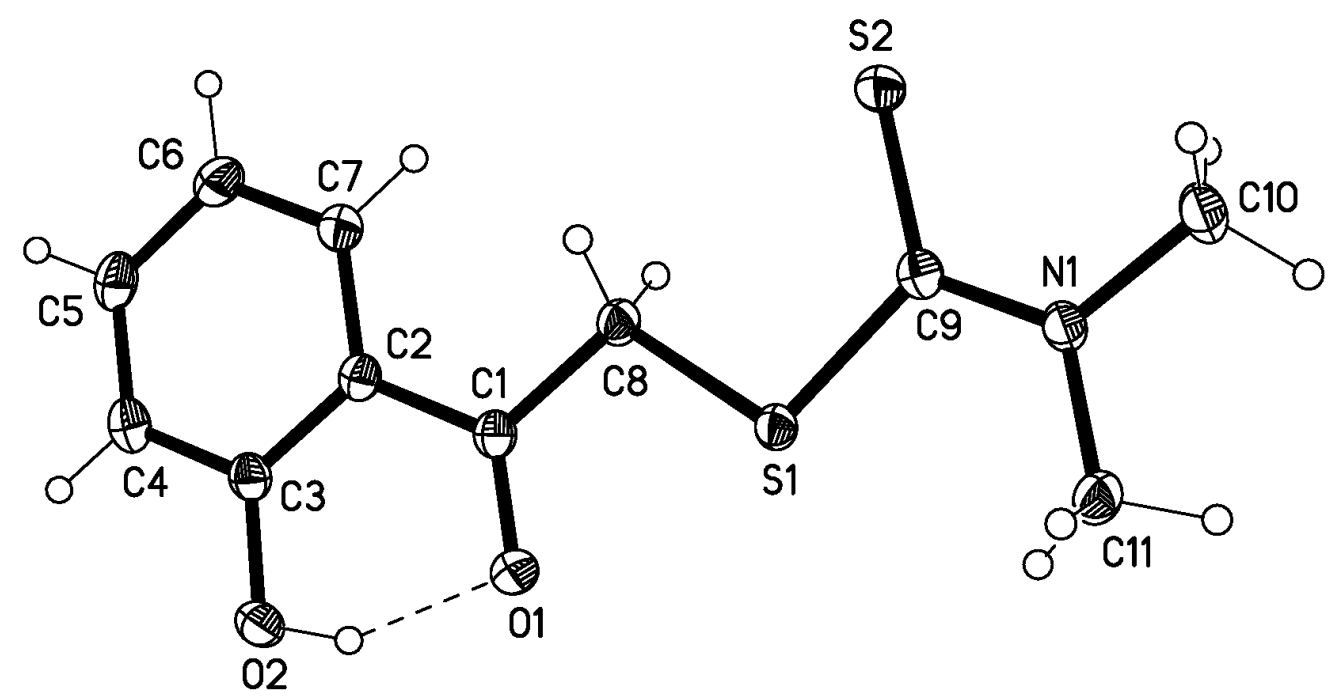

Figure 1. Molecular structure of the dithiocarbamate $\mathbf{2 a}$; ellipsoids represent $50 \%$ probability levels. ${ }^{18}$

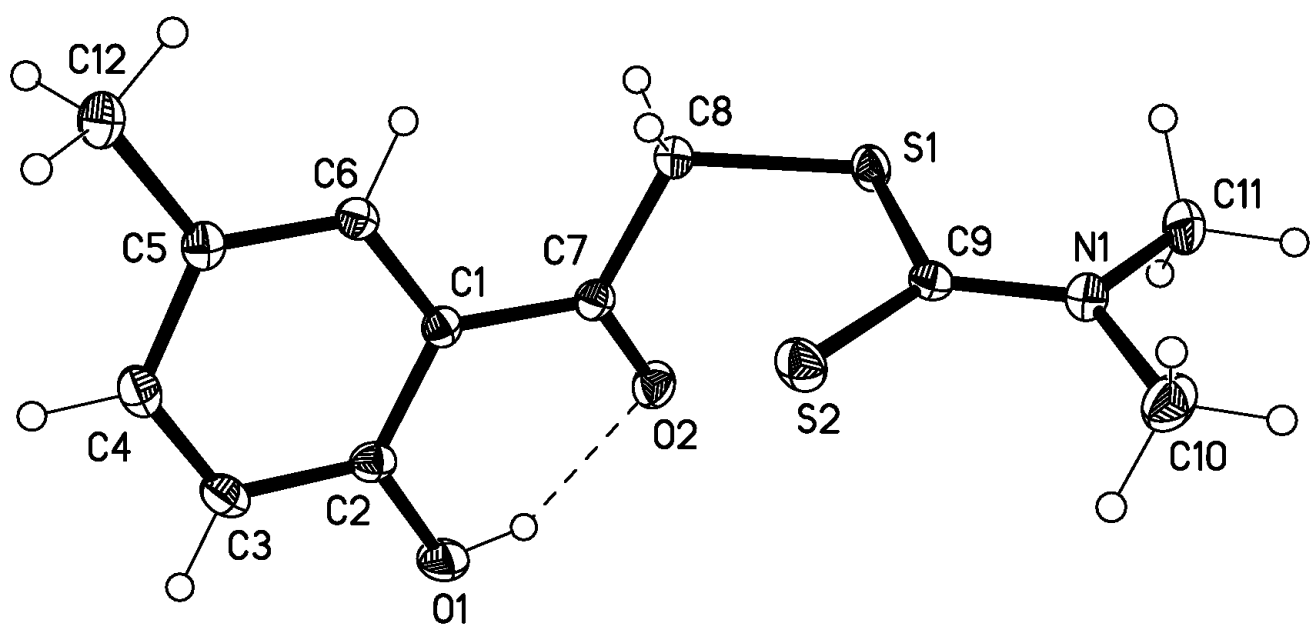

Figure 2. Molecular structure of the dithiocarbamate $\mathbf{2 b}$. Ellipsoids represent $50 \%$ probability levels. ${ }^{19}$

\section{Conclusions}

1,3-Dithiolium ring opening in the presence of excess methylamine is reported. This is an unprecedented reaction since 1,3-dithiol-2-imines are usually formed. The phenacyl dithiocarbamates obtained are identical with those obtained by alternative methods. The structures of the two products were confirmed by $\mathrm{X}$-ray structural analysis. This reaction may serve as a model for ring opening of other cyclic cations of this type. 


\section{Experimental Section}

Melting points: $\mathrm{KSPI}$ melting-point meter. IR: Bruker Tensor $27 .{ }^{1} \mathrm{H}$ and ${ }^{13} \mathrm{C} \mathrm{NMR}$ : Bruker DRX 400 in $\mathrm{CDCl}_{3}$ with tetramethylsilane (TMS) as internal standard at room temp. Chemical shifts are reported in ppm downfield from TMS. MS: Thermo Scientific ISQ LT. Elemental analyses (C, H, N, S) were conducted using a CE440 Elemental Analyzer; the results were found to be in good agreement $( \pm 0.30 \%)$ with the calculated values. All reagents were commercially available and used without further purification.

Reaction of 1,3-dithiolium perchlorates 1a-j with methyl amine. 1-(5-Bromo-2-hydroxy-3-methylphenyl)-1oxoethan-2-yl-N,N-dimethyldithiocarbamate (2d)

General procedure. A solution of methylamine $(1.2 \mathrm{~mL}, 33 \%$ in ethanol, $10 \mathrm{mmol})$ was added to a suspension of 4-(5-bromo-2-hydroxy-3-methylphenyl)-2-( $N, N$-dimethylamino)-1,3-dithiol-2-ylium perchlorate (1d, $0.86 \mathrm{~g}$, $2 \mathrm{mmol}$ ) in ethanol $(15 \mathrm{~mL})$. The reaction mixture was stirred at room temperature until a homogeneous solution was obtained (ca. $2 \mathrm{~h}$ ). After completion of the reaction, the mixture was poured into water $(50 \mathrm{~mL})$ and acidified with hydrochloric acid. The precipitate thus formed was removed by filtration, washed with water, dried and recrystallized from ethanol to give the dithiocarbamate $\mathbf{2 d}(0.29 \mathrm{~g}, 42 \%)$ as a colorless solid. M.p.: $157-158{ }^{\circ} \mathrm{C}$ (lit. $157-158{ }^{\circ} \mathrm{C}$ ). ${ }^{16}$ IR-ATR: $v_{\max }$ 2938, 1640, 1461, 1277, 1109, 989, 747, 620, $529 \mathrm{~cm}^{-1} .{ }^{1} \mathrm{H}$ NMR $\left(400 \mathrm{MHz}, \mathrm{CDCl}_{3}\right): \delta 2.26(3 \mathrm{H}, \mathrm{s}), 3.48(3 \mathrm{H}, \mathrm{s}), 3.56(3 \mathrm{H}, \mathrm{s}), 4.87(2 \mathrm{H}, \mathrm{s}), 7.48(1 \mathrm{H}, \mathrm{d}, 4 \mathrm{~J} 1.3 \mathrm{~Hz}), 7.95(1 \mathrm{H}, \mathrm{d}$, 4J $1.3 \mathrm{~Hz}), 12.08(1 \mathrm{H}, \mathrm{s}) .{ }^{13} \mathrm{C} \mathrm{NMR}\left(100 \mathrm{MHz}, \mathrm{CDCl}_{3}\right): \delta 15.5$ (q), 41.8 (q), 44.6 (t), 46.0 (q), 110.2 (s), 119.5 (s), 129.7 (d), $130.3(\mathrm{~s}), 139.9$ (d), 159.9 (s), 194.8 (s), 198.2 (s) ppm. El-MS m/z (\%): 347 (21) [M , for $\left.\mathrm{C}_{12} \mathrm{H}_{14}{ }^{79} \mathrm{BrNO}_{2} \mathrm{~S}_{2}\right]$.

Dithiocarbamates $\mathbf{2 a}-\mathbf{c}$ and $\mathbf{2 e - j}$ were obtained following the same experimental procedure.

\section{Acknowledgements}

M.L.B. is indebted to the Alexander von Humboldt Foundation for a stay in Braunschweig. CNCS - UEFISCDI support within PNCDI III is acknowledged by LGS and LGB for project numbers PN-III-PI-I.1-PD-2016-0962 and PN-III-PI-I.1-PD-2016-1117, respectively.

\section{Supplementary Material}

Supplementary material containing X-ray crystallographic data for dithiocarbamates $\mathbf{2 a}$ and $\mathbf{2 b}$ can be found in the online version.

\section{References}

1. Yamada, J.-I. Nishikawa, H.; Kikuchi, K. J. Mater. Chem. 1999, 9, 617. https://doi.org/10.1039/a807698g

2. Bryce, M. R. J. Mater. Chem. 1999, 10, 589.

https://doi.org/10.1039/a908385e 
3. Prinzbach. H.; Futterer, E. Adv. Heterocycl. Chem. 1966, 7, 39.

4. Lozach, N.; Stavaux, M. Adv. Heterocycl. Chem. 1980, 27, 152.

5. Lorcy, D.; Robert, A.; Triki, S.; Ouahab, L.; Robin, P.; Chastaing, E. Tetrahedron Lett. 1992, $33,7341$. https://doi.org/10.1016/S0040-4039(00)60182-X

6. Lorcy, D.; Robert, A.; Carlier, R.; Tallec, A. Bull. Soc. Chim. Fr. 1994, 131, 774.

7. Sarbu, L. G.; Lungu, N. C.; Sandu, I. Rev. Chim. (Bucharest) 2019, 70, 745.

8. Birsa, M. L. Synth. Commun. 2003, 33, 3071. https://doi.org/10.1081/SCC-120022483

9. Liang, F.; Li, D.; Zhang, L.; Gao, J.; Liu, Q. Org. Lett. 2007, 9, 4845. https://doi.org/10.1021/ol7021752

10. Ghosh, A. C.; Reinhardt, J. K.; Kindermann, M. K.; Schulzke, C. Chem. Commun. 2014, 50, 10102. https://doi.org/10.1039/C4CC04414B

11. Reisch, J.; Usifoh, C.O.; Oluwadiya, J. O. Monatsh. Chem. 1992, 123, 247. https://doi.org/10.1007/BF00810472

12. Azizi, N.; Akbari, E.; Ebrahimi.; Saidi, M. R. Monatsh. Chem. 2010, 141, 323. https://doi.org/10.1007/s00706-010-0261-0

13. Sarbu, L. G.; Lungu, N. C.; Forna, N. C.; Birsa, M. L. Rev. Chim. (Bucharest) 2013, 64, 1404.

14. Ciobanu, A. S.; Goanta, M.; Birsa, A.; Asaftei, I. V.; Birsa, M. L. Acta Chem. lasi 2008, 16, 61.

15. Lungu, N. C.; Sandu, I.; Chirita, P.; Birsa, M. L. Rev. Chim. (Bucharest) 2013, 64, 697.

16. Birsa, M. L. An. St. Univ. Al.I. Cuza lasi 1998, 6, 57.

17. Goanta, M.; Ciobanu, A. S.; Birsa, A.; Asaftei, I. V.; Birsa, M. L. Acta Chem. lasi 2009, 17, 35.

18. CCDC-1896758 contains the supplementary crystallographic data for compound $\mathbf{2 a}$. These data can be obtained free of charge from The Cambridge Crystallographic Data Centre via www.ccdc.cam.ac.uk/data_request/cif.

19. CCDC-1896757 contains the supplementary crystallographic data for compound $\mathbf{2} \mathbf{b}$. These data can be obtained free of charge from The Cambridge Crystallographic Data Centre via www.ccdc.cam.ac.uk/data request/cif . 